Author affiliations and support information (if applicable) appear at the end of this article.

Published at jco.org on November 2, 2017

Clinical trial information: NCT00774826.

Corresponding author: Stefano Luminari, MD, Hematology, Arcispedale S. Maria

Nuova IRCCS, University of Modena and Reggio Emilia, Viale Risorgimento 80, 42123 Reggio Emilia, Italy; e-mail: stefano.luminari@unimore.it.

(C) 2017 by American Society of Clinical Oncology

$0732-183 X / 18 / 3607 w-689 w / \$ 20.00$

\title{
Long-Term Results of the FOLL05 Trial Comparing R-CVP Versus R-CHOP Versus R-FM for the Initial Treatment of Patients With Advanced-Stage Symptomatic Follicular Lymphoma
}

Stefano Luminari, Angela Ferrari, Martina Manni, Alessandra Dondi, Annalisa Chiarenza, Francesco Merli, Chiara Rusconi, Vittoria Tarantino, Alessandra Tucci, Umberto Vitolo, Sofia Kovalchuk, Emanuele Angelucci, Alessandro Pulsoni, Luca Arcaini, Francesco Angrilli, Gianluca Gaidano, Caterina Stelitano, Giovanni Bertoldero, Nicola Cascavilla, Flavia Salvi, Andrés J.M. Ferreri, Daniele Vallisa, Luigi Marcheselli, and Massimo Federico

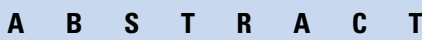

\section{Purpose}

The FOLL05 trial compared R-CVP (rituximab plus cyclophosphamide, vincristine, and prednisone) with R-CHOP (rituximab plus cyclophosphamide, doxorubicin, vincristine, and prednisone) and R-FM (rituximab plus fludarabine and mitoxantrone) regimens without rituximab maintenance as initial therapy for patients with advanced-stage follicular lymphoma (FL). A previous analysis with a median follow-up of 34 months showed a superior 3-year time to treatment failure, the primary study end point, with R-CHOP and R-FM versus R-CVP and showed R-CHOP to have a better risk-benefit ratio in terms of toxicity than R-FM. We report a post hoc analysis of this trial after a median follow-up of 7 years.

\section{Patients and Methods}

Of the 534 enrolled patients, 504 were evaluable. At the time of analysis, the median follow-up was 84 months (range, 1 to 119 months).

\section{Results}

The 8-year time to treatment failure and progression-free survival rates were $44 \%(95 \% \mathrm{Cl}, 39 \%$ to $49 \%)$ and $48 \%(95 \% \mathrm{Cl}, 43 \%$ to $53 \%)$, respectively. The hazard ratio for progression-free survival adjusted by FL International Prognostic Index 2 versus R-CVP was 0.73 for R-CHOP $(95 \% \mathrm{Cl}, 0.54$ to $0.98 ; P=.037)$ and 0.67 for R-FM $(95 \% \mathrm{Cl}, 0.50$ to $0.91 ; P=.009)$. The 8 -year overall survival (OS) rate was $83 \%(95 \% \mathrm{Cl}, 79 \%$ to $87 \%)$, with no significant differences among study arms. Overall, we observed a higher risk of dying as a result of causes unrelated to lymphoma progression with R-FM versus R-CVP.

\section{Conclusion}

With an 83\% 8-year OS rate, long-term follow-up of the FOLL05 trial confirms the favorable outcome of patients with advanced-stage FL treated with immunochemotherapy. The three study arms had similar OS but different activity and toxicity profiles. Patients initially treated with R-CVP had a higher risk of lymphoma progression compared with those receiving $\mathrm{R}-\mathrm{CHOP}$, as well as a higher risk of requiring additional therapy.

\section{J Clin Oncol 36:689-696. (C) 2017 by American Society of Clinical Oncology}

\section{ASSOCIATED CONTENT}

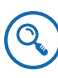
Appendix DOI: https://doi.org/10.1200/JCO 2017.74.1652

(Q) Data Supplement DOI: https://doi.org/10.1200/JCO 2017.74.1652

DOI: https://doi.org/10.1200/JCO.2017 74.1652

\section{INTRODUCTION}

Follicular lymphoma (FL) is the most common of the indolent non-Hodgkin lymphomas (NHLs) and constitutes approximately $10 \%$ to $20 \%$ of all newly diagnosed NHLs in Western countries. ${ }^{1}$ The natural history of FL is that of a low-grade lymphoma, with the typical pattern of relapses after initial therapy followed by remissions of increasingly shorter duration after each event and by the risk of transformation into aggressive lymphoma. ${ }^{2}$ With the availability of anti-CD20 monoclonal antibody, the outcome for patients with FL has greatly improved, and sequential treatment with active immunochemotherapy (ICT) regimens is associated with high response rates and prolonged survival. ${ }^{3}$ Patients with FL now 
show remission rates of nearly $90 \%$, a median progression-free survival (PFS) of 6 to 7 years, and 5-year overall survival (OS) rates approaching $90 \%$. $^{4-6}$

Although the use of anti-CD20 monoclonal antibody is confirmed by strong evidence, choice of chemotherapy backbone has been a matter of debate for many years and is still made based on limited evidence. In 2005, the Fondazione Italiana Linfomi initiated the randomized FOLL05 trial to identify the best ICT regimen for first-line treatment of advanced-stage FL among R-CVP (rituximab plus cyclophosphamide, vincristine, and prednisone), R-CHOP (rituximab plus cyclophosphamide, doxorubicin, vincristine, and prednisone), and R-FM (rituximab plus fludarabine and mitoxantrone). In 2013, we published the primary analysis of the study, with a median follow-up of 34 months, showing the superiority of R-CHOP and R-FM over R-CVP in terms of time to treatment failure (TTF) and PFS, but our results also revealed a better toxicity profile for R-CHOP compared with R-FM. ${ }^{7}$ Overall, these data were interpreted to suggest R-CHOP as the standard ICT for the treatment of patients with advanced-stage FL.

The use of early end points as surrogates of OS is accepted by the scientific community and enables clinical trials of indolent disease. However, the long natural history of FL warrants a longterm update of clinical trials to better evaluate the risk-benefit ratio of treatment. Here, we present an analysis of the mature results of the FOLL05 trial after a median follow-up of 7 years.

\section{PATIENTS AND METHODS}

\section{Patients}

FOLL05 was a prospective, randomized, open-label, multicenter phase III trial that included previously untreated patients with advancedstage symptomatic FL. Eligible patients had a histologically confirmed diagnosis of grade 1, 2, or 3a FL according to the 2008 WHO classification, ${ }^{8}$ had Ann Arbor stage II to IV disease, were age 18 to 75 years with Eastern Cooperative Oncology Group performance status of 0 to 2, and had active disease according to Italian Society of Hematology guidelines. ${ }^{9}$ Exclusion criteria included a diagnosis of grade $3 \mathrm{~b}$ FL, evidence of histologic transformation into an aggressive lymphoma at the time of diagnosis, CNS involvement, or history of previous malignancy.

This study was conducted in compliance with the Declaration of Helsinki and in accordance with Good Clinical Practice rules and was approved by a research ethics committee. All enrolled patients provided written informed consent. Patients were randomly assigned to receive eight doses of rituximab combined with eight courses of CVP (arm A [R-CVP]), six cycles of CHOP (arm B [R-CHOP]), or six cycles of FM (arm C [R-FM]). Because its cost was not reimbursed in 2005, maintenance was not admitted and was considered to be failure for the primary study end point if administered.

Response was assessed with clinical examination, contrastenhanced computed tomography, and bone marrow biopsy if required. $\left[{ }^{18} \mathrm{~F}\right]$ fluorodeoxyglucose positron emission tomography was not mandatory for response assessment but was performed in a significant proportion of patient cases. ${ }^{10}$ When available, $\left[{ }^{18} \mathrm{~F}\right]$ fluorodeoxyglucose positron emission tomography was not considered for staging or definition of response. Clinical examination of patients who completed treatment was planned every 3 months for the first 6 months, every 6 months for 3 years, and then annually. Computed tomography scans were performed every 6 months for 2 years, then annually or when clinically indicated. Follow-up updates were actively conducted among participating institutions. Relapses or progressions were determined based on clinical or radiologic assessment.

\section{Statistical Methods}

In addition to TTF, the primary study end point, this long-term analysis was conducted to evaluate PFS, OS, cause-specific mortality (CSM), cumulative incidence of second malignancies (SMs), and frequency of late adverse events. For this analysis, the initial definition of sample size was not applicable; the analysis was conducted as a post hoc long-term observational study of patients enrolled in the FOLL05 trial. Molecular response was also included as a secondary end point and results published in a separate report. ${ }^{11}$

TTF was defined as the time from random assignment to discontinuation of treatment of any reason, including disease progression, treatment toxicity, start of maintenance therapy, or death. PFS was defined as the time from random assignment to progressive disease or death resulting from any cause. OS was calculated as the time from patient random assignment to death resulting from any cause. Adverse events were registered in accordance with the standard Common Toxicity Criteria for Adverse Events (version 3).

For the analysis of CSM, cause of death was described as reported by the local investigator and classified as lymphoma related in the case of documented uncontrolled progression of lymphoma or non-lymphoma related (NLR) in patients who died as a result of causes not directly related to lymphoma progression. NLR events were additionally classified as resulting from SMs or other causes. Death resulting from SM was considered in the case of death directly associated with the presence of an uncontrolled and/or progressive SM or occurring during treatment for SM; death resulting from causes other than lymphoma progression or SM was classified as resulting from other causes.

Survival curves were calculated using the Kaplan-Meier method and compared using the log-rank test. Effect sizes were reported as hazard ratios (HRs) with 95\% CIs and estimated using the Cox proportional hazards regression method, adjusted by relevant confounding factors when needed. Risk of SM was reported as a cumulative incidence function, with death as a competing risk, using the method of Gooley et al ${ }^{12}$; comparisons between curves were performed using the Gray test ${ }^{13}$ and Fine-Gray regression. ${ }^{14}$ The cumulative risk of histologic transformation and CSM was reported according to the Nelson-Aalen estimator. For the purposes of this study, only biopsy-proven histologic transformations were considered.

All reported tests were two sided, and $P<.05$ was considered to indicate moderate strength of evidence against the null hypothesis. $P$ values were not adjusted for multiple comparisons. The analysis was performed according to the intention-to-treat (ITT) approach, except for studies involving SMs and late adverse events, which were analyzed according to actual therapy received.

\section{RESULTS}

From March 2006 to September 2010, 534 patients were enrolled in the FOLL05 trial by 58 Italian institutions; of these patients, 504 were eligible for ITT analysis (Fig 1). The main characteristics of eligible patients, whose median age was 55 years (range, 30 to 75 years), were described in the original report. ${ }^{7}$

The median follow-up was 84 months (range, 1 to 119 months). Overall, 43 patients were lost to follow-up (8.5\%) after a median time of 64 months (range, 1 to 101 months). Minimum follow-up for surviving patients not lost to follow-up was 4.5 years. With prolonged follow-up, the 8-year TTF was 44\% (95\% CI, 39\% to 49\%). R-CHOP and R-FM had better TTF rates than R-CVP: $45 \%$ (HR, 0.73; 95\% CI, 0.55 to $0.98 ; P=.033)$ and $49 \%(\mathrm{HR}, 0.70$; $95 \%$ CI, 0.52 to $0.93 ; P=.016)$ versus $38 \%$ at 8 years, respectively (Fig 2A).

Overall, 252 events were recorded for PFS, including 68 additional episodes compared with the initial report (five progressions, 


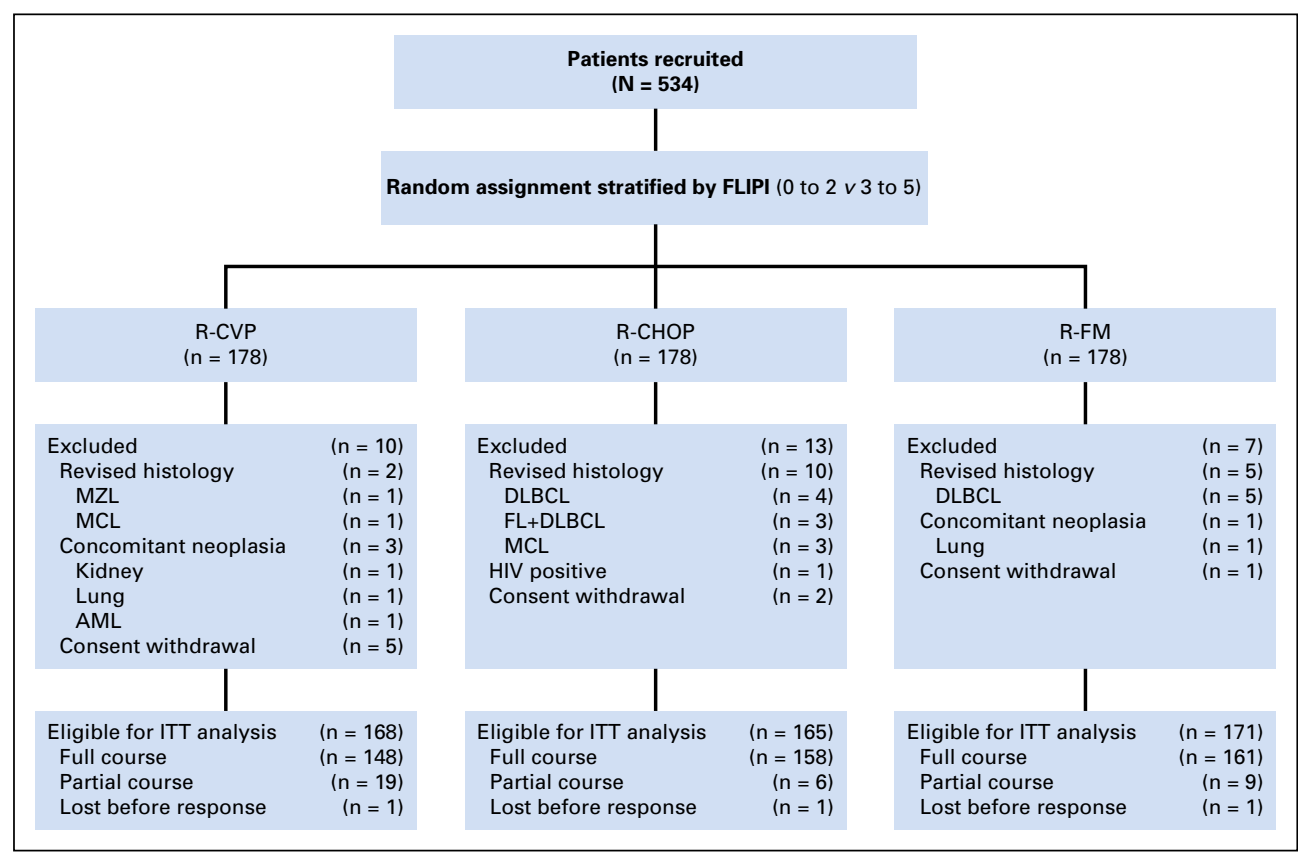

Fig 1. Treatment allocation and No. of patients included in the analysis. AML, acute myeloid leukemia; $\mathrm{DLBCL}$, diffuse large B-cell lymphoma; FL, follicular lymphoma; FLIPI, Follicular Lymphoma International Prognostic Index; ITT, intention to treat; $\mathrm{MCL}$, mantle cell lymphoma; MZL, marginal zone lymphoma; R-CHOP, rituximab plus cyclophosphamide, doxorubicin, vincristine, and prednisone; R-CVP, rituximab plus cyclophosphamide, vincristine, and prednisone; R-FM, rituximab plus fludarabine and mitoxantrone.

58 relapses, and five deaths not related to lymphoma progression; Table 1). The 8 -year PFS rate was $48 \%$ (95\% CI, $43 \%$ to $52 \%$ ); for patients randomized to the R-CVP, R-CHOP, and R-FM arms, it was $42 \%$ (95\% CI, $35 \%$ to $50 \%$ ), $49 \%$ (95\% CI, $40 \%$ to $57 \%$ ), and $52 \%$ (95\% CI, 45\% to 60\%), respectively (Fig 2B). Considering PFS adjusted by the FL International Prognostic Index 2, the HRs between R-CHOP versus R-CVP and R-FM versus R-CVP were 0.73 (95\% CI, 0.54 to $0.98 ; P=.037)$ and 0.67 (95\% CI, 0.50 to $0.91 ; P=.009)$.

\section{Salvage Therapy}

Overall, 208 of 248 patients who had primary refractory or experienced progressive or relapsed disease required salvage treatment: 90 received conventional ICT, 75 underwent autologous stem-cell transplantation (ASCT), 33 were treated with immunotherapy alone, and 10 were treated with radiotherapy alone (Table 2).

Among the 75 patients who underwent ASCT, a similar distribution by study arm was observed $(n=27,27$, and 21 for

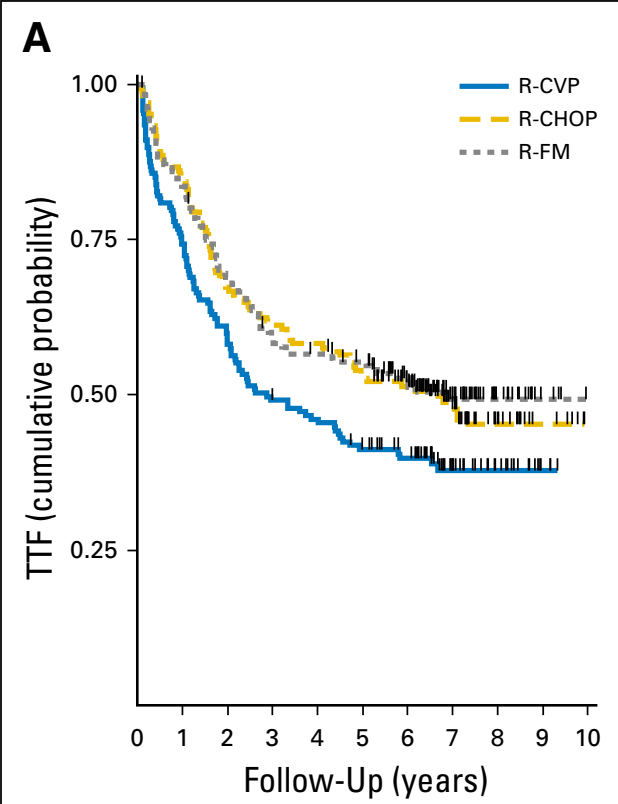

No. at risk:

$\begin{array}{llllllllllll}\text { R-CVP } & 168 & 124 & 97 & 81 & 76 & 66 & 56 & 27 & 15 & 3 & 0\end{array}$ $\begin{array}{llllllllllll}\text { R-CHOP } & 165 & 140 & 111 & 101 & 96 & 87 & 73 & 44 & 18 & 7 & 0\end{array}$

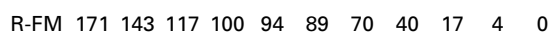

\section{B}

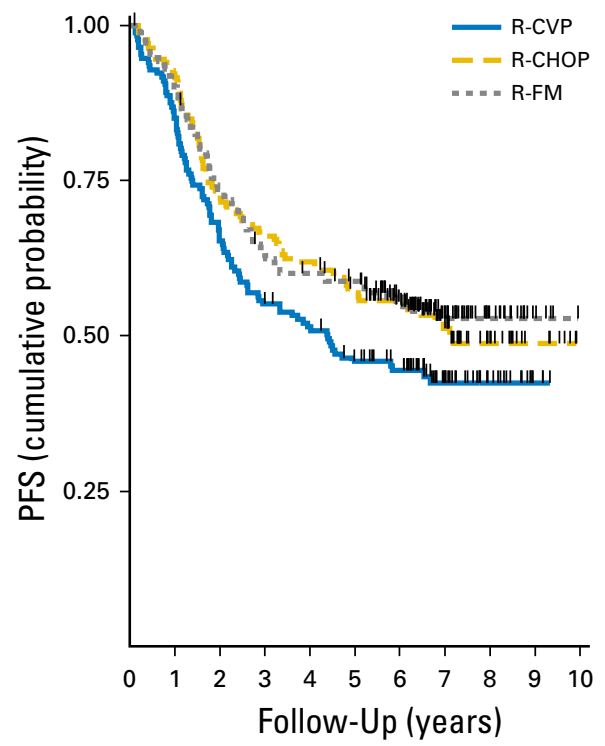

No. at risk:

$\begin{array}{llllllllllll}\text { R-CVP } & 168 & 142 & 109 & 91 & 84 & 72 & 62 & 31 & 16 & 3 & 0\end{array}$

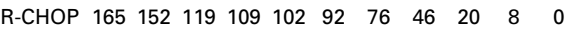

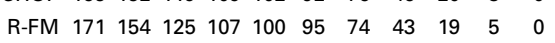

Fig 2. (A) Time to treatment failure (TTF; $\mathrm{R}-\mathrm{CHOP}$ [rituximab plus cyclophosphamide, doxorubicin, vincristine, and prednisone] $v$ R-CVP [rituximab plus cyclophosphamide, vincristine, and prednisone]: $P=.033$; R-FM [rituximab plus fludarabine and mitoxantrone] $v$ R-CVP: $P=.016)$ and (B) progression-free survival (PFS) by treatment arm (R-CHOP $v$ R-CVP adjusted by Follicular Lymphoma International Prognostic Index 2 [FLIPI-2]: $P=.037$; R-FM $v$ R-CVP adjusted by FLIPI-2: $P=.009$ ). 


\begin{tabular}{|c|c|c|c|c|c|c|c|c|c|c|}
\hline \multirow[b]{3}{*}{ Event } & \multicolumn{9}{|c|}{ ITT } & \multirow[b]{3}{*}{ Total } \\
\hline & \multicolumn{3}{|c|}{$\begin{array}{c}\text { R-CVP } \\
(n=168)\end{array}$} & \multicolumn{3}{|c|}{$\begin{array}{l}\text { R-CHOP } \\
(\mathrm{n}=165)\end{array}$} & \multicolumn{3}{|c|}{$\begin{array}{c}\text { R-FM } \\
(\mathrm{n}=171)\end{array}$} & \\
\hline & Early & Update & Total & Early & Update & Total & Early & Update & Total & \\
\hline \multicolumn{11}{|l|}{ PFS } \\
\hline Progression & 3 & - & 3 & 4 & - & 4 & 6 & - & 6 & 13 \\
\hline Progression after SD & 7 & 2 & 9 & 2 & 1 & 3 & 2 & 2 & 4 & 16 \\
\hline Relapse from CR/PR & 64 & 16 & 80 & 44 & 25 & 69 & 44 & 17 & 61 & 210 \\
\hline Death resulting from any cause & - & 2 & 2 & 2 & 2 & 4 & 6 & 1 & 7 & 13 \\
\hline Total & 74 & 20 & 94 & 52 & 28 & 80 & 58 & 20 & 78 & 252 \\
\hline \multicolumn{11}{|l|}{ Cause of death } \\
\hline FL progression & 4 & 13 & 17 & 6 & 8 & 14 & 8 & 7 & 15 & 46 \\
\hline SM & - & 1 & 1 & 1 & 3 & 4 & 4 & 3 & 7 & 12 \\
\hline Cardiopathy & - & 1 & 1 & 1 & 1 & 2 & 2 & - & 2 & 5 \\
\hline Sepsis & - & - & - & - & 1 & 1 & 2 & 1 & 3 & 4 \\
\hline GVHD liver & - & - & - & - & - & - & - & 1 & 1 & 1 \\
\hline Cachexia & - & - & - & 1 & - & 1 & - & - & - & 1 \\
\hline Car accident & - & - & - & 1 & - & 1 & - & - & - & 1 \\
\hline Hemorrhage & - & - & - & - & - & - & 1 & - & 1 & 1 \\
\hline Intestinal infarction & - & - & - & - & - & - & - & 1 & 1 & 1 \\
\hline Unknown & - & 2 & 2 & - & - & - & - & 1 & 1 & 1 \\
\hline Total & 4 & 17 & 21 & 10 & 13 & 23 & 17 & 14 & 31 & 75 \\
\hline
\end{tabular}

R-CVP, R-CHOP, and R-FM, respectively). Overall, patients initially randomly assigned to R-CVP were at higher risk for requiring a second-line therapy (91 patients [55\%]), compared with those receiving $\mathrm{R}-\mathrm{CHOP}(\mathrm{n}=63$ [38\%]) or $\mathrm{R}-\mathrm{FM}(\mathrm{n}=54$ [32\%]; $\mathrm{P}<.001)$. Patients treated with R-CVP had $43 \%$ higher probability of requiring salvage therapy versus those receiving $\mathrm{R}-\mathrm{CHOP}$.

\section{SMs}

During follow-up, 41 SMs were reported: 14 were hematologic and 27 were solid cancers. Twenty-one SMs were diagnosed in patients who never experience relapse and never received salvage therapy ( $\mathrm{n}=1,10$, and 10 for R-CVP, R-CHOP, and R-FM, respectively; Table 3 ). The cumulative incidence of SMs at 8 years was $9.4 \%(95 \% \mathrm{CI}, 6.8 \%$ to $13.0 \%)$, and the median time to SM development was 33 months (range, 8 to 96 months). The cumulative incidence by actual treatment at 8 years was $6.2 \%(95 \%$ CI, $2.5 \%$ to $14.8 \%$ ), $12 \%$ (95\% CI, $7.7 \%$ to $18.7 \%$ ), and $9.6 \%$ (95\% CI, $6.0 \%$ to $15.2 \%$ ) for R-CVP, R-CHOP, and R-FM, respectively (Gray test $P=.077$ ). The HRs for SMs between R-CHOP and R-CVP and between R-FM and R-CVP were 2.59 (95\% CI, 1.09 to $6.20 ; P=.032$ ) and $2.29(95 \% \mathrm{CI}, 0.94$ to $5.56 ; P=.067)$,

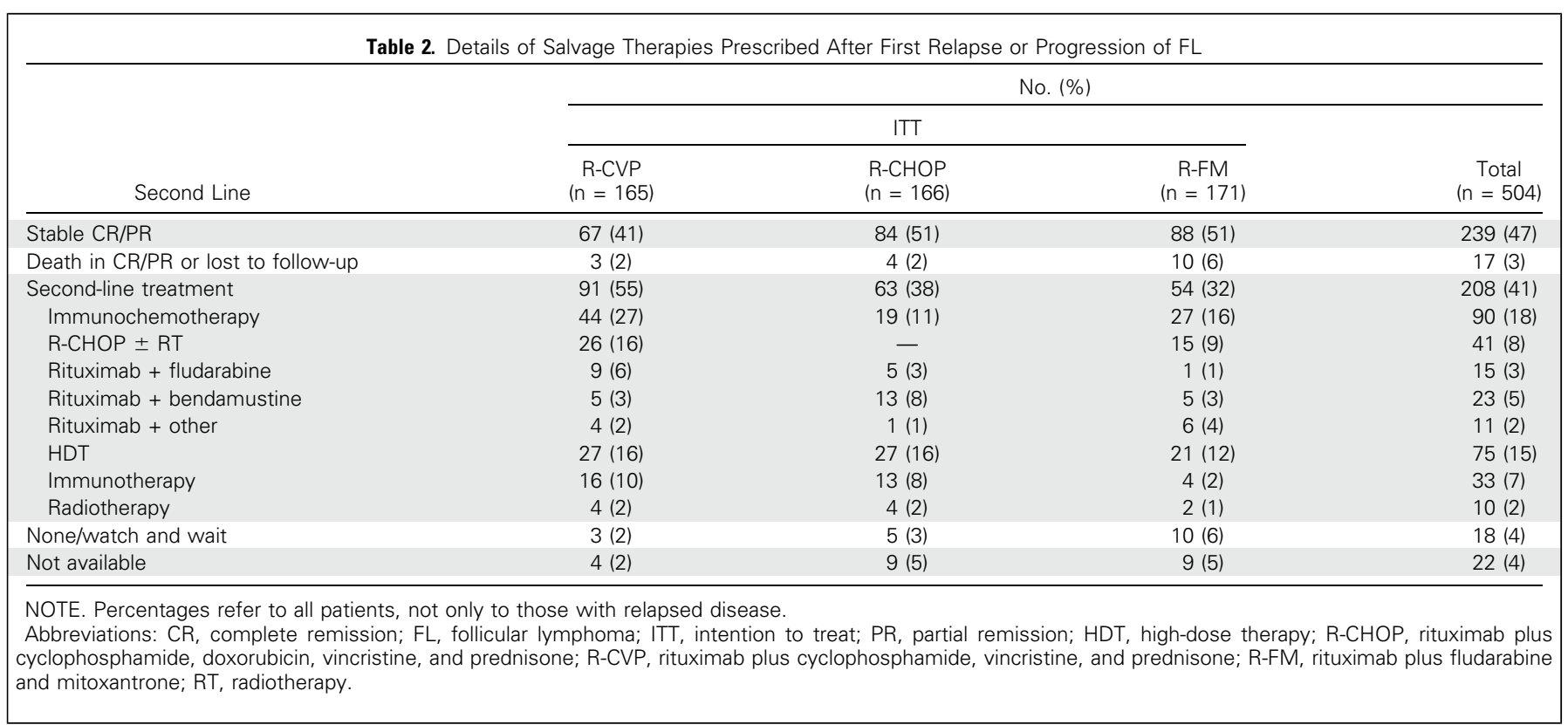


Table 3. SMs by Treatment Arm for All Patients in FOLL05 Trial and for Those Without Relapsed Disease or Salvage Therapy

\begin{tabular}{|c|c|c|c|c|c|c|c|c|}
\hline \multirow[b]{3}{*}{ SM } & \multicolumn{8}{|c|}{ Actual Treatment } \\
\hline & \multicolumn{4}{|c|}{ No Relapse or Salvage Therapy } & \multicolumn{4}{|c|}{ All Patients in Study } \\
\hline & $\begin{array}{l}\text { R-CVP } \\
(n=69)\end{array}$ & $\begin{array}{l}\text { R-CHOP } \\
(n=87)\end{array}$ & $\begin{array}{c}\text { R-FM } \\
(n=98)\end{array}$ & $\begin{array}{c}\text { Total } \\
(n=254)\end{array}$ & $\begin{array}{c}\text { R-CVP } \\
(n=165)\end{array}$ & $\begin{array}{l}\mathrm{R}-\mathrm{CHOP} \\
(\mathrm{n}=166)\end{array}$ & $\begin{array}{c}\text { R-FM } \\
(n=171)\end{array}$ & $\begin{array}{c}\text { Total } \\
(n=502)\end{array}$ \\
\hline Breast cancer (female) & - & 1 & - & 1 & 1 & 2 & 1 & 4 \\
\hline Uterine cancer & - & 1 & $2\left(1^{*}\right)$ & $3(1 *)$ & - & 1 & $2\left(1^{*}\right)$ & $3(1 *)$ \\
\hline Prostate cancer & - & 1 & 1 & 2 & 2 & 1 & 1 & 4 \\
\hline Lung cancer & - & $2(1 *)$ & $1 *$ & $3(2 *)$ & - & $2(1 *)$ & $1 *$ & $3(2 *)$ \\
\hline Kaposi sarcoma/skin cancer & - & - & 1 & 1 & 1 & 1 & 2 & 4 \\
\hline GI tract & - & 1 & - & 1 & 1 & 1 & $1 *$ & $3(1 *)$ \\
\hline Urothelial cancer & - & 2 & - & 2 & - & 2 & - & 2 \\
\hline Pancreatic cancer & - & - & - & - & - & - & $1 *$ & $1^{*}$ \\
\hline Melanoma & 1 & - & 1 & 2 & 1 & - & 1 & 2 \\
\hline Glioblastoma & - & $1 *$ & - & $1^{*}$ & - & $1^{*}$ & - & $1^{*}$ \\
\hline AML/MDS & - & 1 & $3(2 *)$ & $5(2 *)$ & $1^{*}$ & $3\left(2^{*}\right)$ & $5\left(3^{*}\right)$ & $9\left(6^{*}\right)$ \\
\hline Hodgkin lymphoma & - & - & 1 & 1 & - & 1 & 1 & 2 \\
\hline Multiple myeloma & - & - & - & - & - & 1 & - & 1 \\
\hline CLL & - & - & - & - & - & 1 & - & 1 \\
\hline SMZL & - & - & - & - & - & 1 & - & 1 \\
\hline Total & 1 & $10\left(2^{*}\right)$ & $10\left(4^{*}\right)$ & $21(6 *)$ & $7\left(1^{*}\right)$ & $18\left(4^{*}\right)$ & $16\left(7^{*}\right)$ & $41\left(12^{*}\right)$ \\
\hline Histologic transformation & - & - & - & - & $5\left(2^{*}\right)$ & $4\left(3^{*}\right)$ & 2 & $11\left(5^{*}\right)$ \\
\hline
\end{tabular}

respectively. At time of relapse, 81 of 210 patients had undergone biopsy; histologic transformation was documented and biopsy confirmed in 11 patients, with a $2.9 \%$ (95\% CI, $1.5 \%$ to $5.5 \%)$ cumulative incidence at 8 years (Table 3 ).

\section{os}

Overall, 75 deaths were reported: 21,23 , and 31 in the R-CVP, R-CHOP, and R-FM arms, respectively. The 8-year OS rate was $83 \%$ (95\% CI, $78 \%$ to $86 \%$ ); by ITT analysis, it was $85 \%$ (95\% CI, $77 \%$ to $91 \%$ ), $83 \%$ (95\% CI, $75 \%$ to $89 \%$ ), and $79 \%$ (95\% CI, $71 \%$

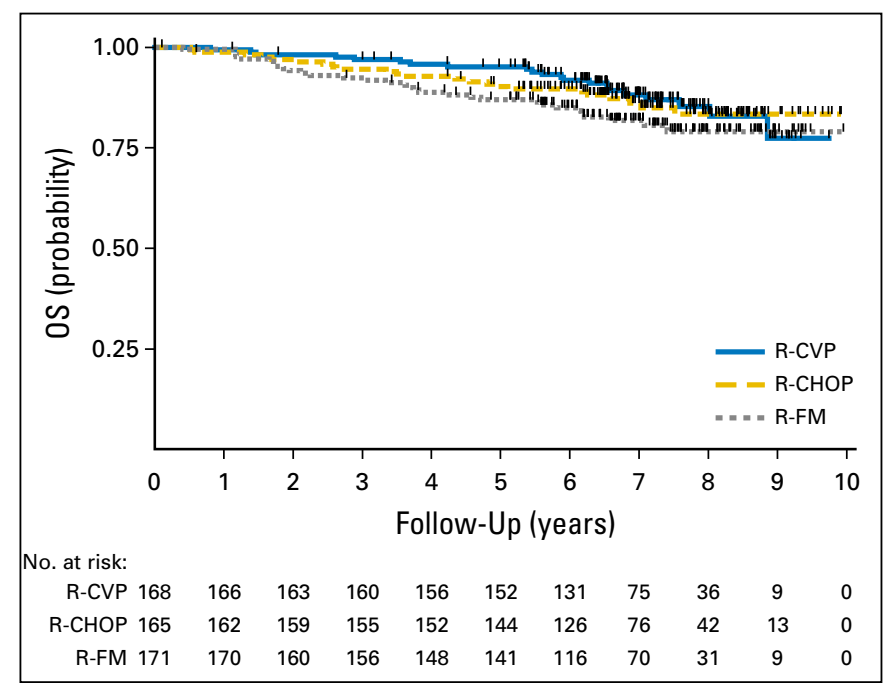

Fig 3. Overall survival (OS) by treatment arm according to intention-to-treat analysis (R-CVP [rituximab plus cyclophosphamide, vincristine, and prednisone] $v$ R-CHOP [rituximab plus cyclophosphamide, doxorubicin, vincristine, and prednisone] $v$ R-FM [rituximab plus fludarabine and mitoxantrone]: log-rank $P=.243$ ). to $85 \%)$ for R-CVP, R-CHOP, and R-FM, respectively $(P=.243$; Fig 3).

Forty-six deaths $(61 \%)$ resulted from lymphoma progression, and $29(39 \%)$ resulted from other causes (Table 1). None of the fatal infectious events occurred during induction therapy or in patients who never experienced relapse. The risk of death resulting from lymphoma was comparable among study arms $(P=.900)$, whereas the risk of death resulting from NLR causes was higher with R-FM ( $11.2 \%$ at 8 years) than with R-CVP $(1.8 \% ; P=.005)$; the NLR CSM difference between R-CVP and R-CHOP (6.4\% for R-CHOP) was not statistically significant $(P=.157$; Figs $4 \mathrm{~A}$ and $4 \mathrm{~B})$. Of the 12 deaths resulting from SMs, seven were reported in the R-FM arm, four in the R-CHOP arm, and one in the R-CVP arm (Table 3); in the only patient treated with R-CVP and in one patient treated with $\mathrm{R}-\mathrm{CHOP}$, an SM was diagnosed after salvage ASCT. The CSM curves by SMs and by other causes are provided in Figures $4 \mathrm{C}$ and 4D.

\section{DISCUSSION}

With our long-term analysis of the FOLL05 trial, we provide new and more detailed data on the randomized comparison among R-CVP, R-CHOP, and R-FM. Overall, the observed 8-year OS rate of $84 \%$ clearly shows that the natural history of patients with FL has improved compared with the past. In addition, a significant proportion of patients ( $48 \%$ in this study) were still free from lymphoma progression 8 years after initial diagnosis, and at least in some patients, this result may translate into a possible cure of lymphoma. Although the study was not originally powered for OS analysis, no differences were observed for OS among the three study arms; however, this result was achieved with the need for second-line therapy in one of four patients treated with R-CVP versus those randomly assigned to R-CHOP and at the cost of higher mortality 

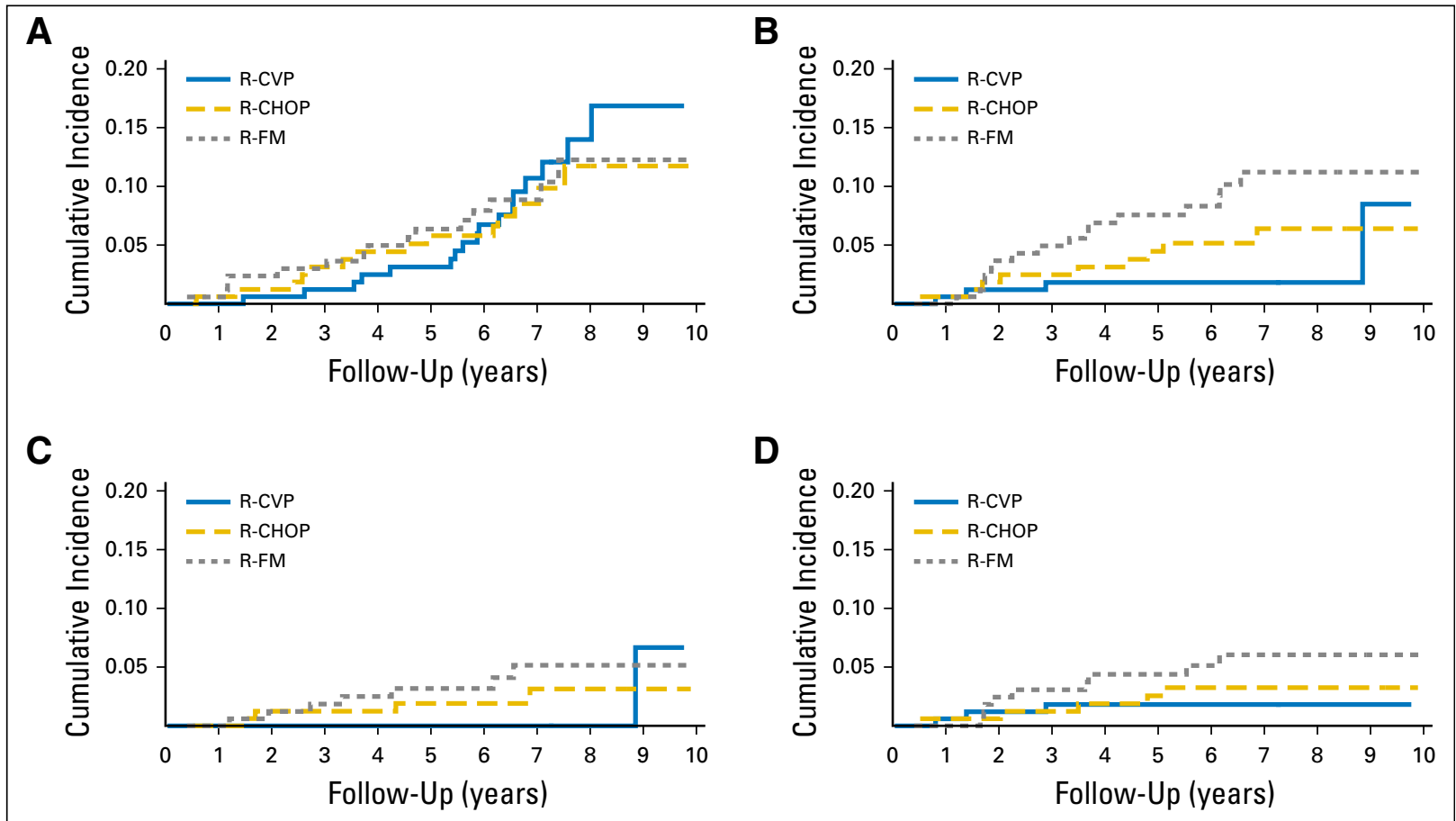

Fig 4. Cumulative incidence of cause-specific mortality stratified by arm. (A) All lymphoma-related causes of death (overall $P=.900)$; (B) all non-lymphoma-related (NLR) causes of death (overall $P=.019$ ); (C) second-cancer NLR causes of death only $(P=.090)$; and (D) all other NLR causes of death, excluding second malignancies $(P=.179)$. $\mathrm{R}$-CHOP, rituximab plus cyclophosphamide, doxorubicin, vincristine, and prednisone; R-CVP, rituximab plus cyclophosphamide, vincristine, and prednisone; R-FM, rituximab plus fludarabine and mitoxantrone.

for causes unrelated to lymphoma observed among patients treated with R-FM compared with R-CVP.

Our results compare favorably with those of similar studies. In the 83 -month observation of the FL2000 study, ${ }^{15}$ the 8 -year eventfree survival and OS rates were $44.1 \%$ and $78.6 \%$ for patients randomly assigned to R-CHVP (cyclophosphamide, adriamycin, vincristine, and prednisone) and interferon. In the OSHO\#39 (East German Study Group of Hematology and Oncology) study, ${ }^{16}$ after a median follow-up of 102 months, the 8-year OS rate was $76.1 \%$, and the median event-free survival was 89.6 months for surviving patients treated with R-MCP (rituximab plus mitoxantrone, chlorambucil, and prednisolone). Finally, the 6-year update from the PRIMA (Primary Rituximab and Maintenance) trial described a 6-year PFS rate of $42.7 \%$ and a favorable 6-year OS rate of $88.7 \%$ for patients randomly assigned to the observation arm. ${ }^{17}$

With this update of the FOLL05 study, we contribute to the definition of standard ICT in the initial treatment of patients with advanced-stage FL. Our CSM data provide a better description of the consequences of late events for patient survival, focusing on life-threatening events and reducing the impact of curable conditions that are also subject to under-reporting. Patients treated with R-FM had high rates of SMs and a higher risk of dying as a result of causes unrelated to lymphoma progression compared with those receiving R-CVP.

The association between fludarabine-based regimens and SMs has been already reported for NHLs. ${ }^{18-22}$ Sacchi et al $^{23}$ described a 12 -year cumulative incidence rate of $10.5 \%$ for 563 patients with indolent NHL enrolled in Gruppo Italiano Studio Linfomi trials from 1988 to 2003. More recently, in a GELCAB (Grup d'Estudi dels Linfomes de Catalunya i Balears) study, the 10-year risk of SM was $8 \%$, similar to what was found in our study, ${ }^{24}$ and the 10 -year rate was high $(27 \%)$ in FND (fludarabine, mitoxantrone, and dexamethasone) -treated patients, as reported by Nastoupil et al. ${ }^{25}$ Although the risk of SM is known with fludarabine combinations, we should acknowledge that FCR (fludarabine, cyclophosphamide, and rituximab) is well regarded as a highly effective and appropriate regimen for patients with chronic lymphocytic lymphoma and is the current standard against which other regimens are compared. With increasing treatment efficacy, the problem of SM is suggested as a relevant issue in FL and has been observed in other lymphoma subtypes similarly treated with rituximab-containing regimens. ${ }^{26-28}$

In our initial report, we suggested that R-CHOP was a better option than R-CVP. Considering the updated results, we conclude with high confidence that patients treated with R-CHOP had a lower risk of progressive disease than those treated with R-CVP. In addition, our analysis of OS suggests that survival is similar between R-CHOP and R-CVP. Regarding the OS data, however, it should be emphasized that because of the small number of events, these results are not sufficiently powered to draw definitive conclusions. In addition, the choice of salvage therapy in patients with progressive disease was not defined in the protocol, and actually, high heterogeneity among second-line therapies was seen (Table 2). Our data suggest that patients initially treated with R-CVP maintained high chances of achieving second remission but also had a $43 \%$ higher risk of requiring second-line treatment compared with those receiving R-CHOP. In particular, when salvage treatment was needed after R-CVP, this included R-CHOP in 26 patient cases $(27 \%$ of relapsed disease cases) or high-dose therapy in 27 patient cases (28\%). Moreover, if durable remission may translate into a so-called cure of FL in a proportion of patients, we can also conclude that 
considering R-CHOP as initial therapy, patients have a higher chance of being cured with the first course of therapy.

To put our results in perspective, we should acknowledge some possible concerns. First, no maintenance therapy was allowed in the FOLL05 trial, and we were unable to estimate the effect of prolonged use of rituximab in the comparison among study arms. Even if it has been shown that the risk of progressive disease is significantly reduced by maintenance rituximab, no data are available to suggest a different activity of maintenance between R-CHOP and R-CVP. Moreover, considering that in the 6-year update of the PRIMA trial, maintenance therapy did not result in any difference in OS, ${ }^{17}$ we assume that our results might also be reproduced under the conditions of maintenance therapy. Second, because the analysis of late events is time dependent, and several events were reported after salvage therapies, longer follow-up would be required.

Third, in recent years, the combination of BR (bendamustine and rituximab) has been rapidly imposed as first-choice therapy for patients with advanced-stage FL in need of treatment and is now widely adopted. ${ }^{29,30}$ BR has been compared with R-CHOP or $\mathrm{R}-\mathrm{CVP}$ in two different trials, showing discordant results in favor of higher antilymphoma activity of the new combination but also showing strong evidence in favor of a better toxicity profile of $\mathrm{BR} .{ }^{27,31}$ Recently, long-term follow-up data from these studies have been presented, confirming activity and safety data of the BR combination; however, similarly to our study, differences in terms of PFS did not translate into OS differences. ${ }^{32,33}$ Thus, given the similar efficacy of BR versus R-CHOP, we believe they both represent the best available options to achieve long-lasting remissions in patients with previously untreated FL. Further improvement is expected with the adoption of maintenance therapy and with the substitution of rituximab with the novel anti-CD20 agent obinotuzumab, which was recently shown to further reduce the risk of progression. $^{34}$

Finally, as ancillary results, we were also able to complete our study with details on the histologically documented transformation of FL into aggressive lymphoma. The observed transformation rate was low compared with those in previous reports from retrospective studies $^{35-37}$ and similarly low compared with the $4.1 \%$ rate at 6 years recently reported from the analysis of the PRIMA trial. ${ }^{38}$ Although a formal and appropriate methodologic demonstration is lacking, a protective effect of rituximab on the rate of transformation can be suggested, as also recently reported by a large retrospective international study. ${ }^{39}$

In conclusion, this long-term update of the FOLL05 trial confirms the high efficacy of ICT for the initial treatment of patients with advanced-stage FL in need of therapy. In addition, with the longer follow-up, we can conclude that if the aim of initial therapy is to maximize treatment activity and increase the chance of durable disease control, R-CHOP should be the preferred option among the three regimens. Conversely, R-CVP might be seen as a good option in patients for whom the goal of therapy is treatment tolerability; in this case, however, patients should be informed about the higher probability of requiring second-line treatment.

\section{AUTHORS' DISCLOSURES OF POTENTIAL CONFLICTS OF INTEREST}

Disclosures provided by the authors are available with this article at jco.org.

\section{AUTHOR CONTRIBUTIONS}

Conception and design: Stefano Luminari, Massimo Federico Provision of study materials or patients: Stefano Luminari, Annalisa Chiarenza, Francesco Merli, Alessandra Tucci, Umberto Vitolo, Emanuele Angelucci, Alessandro Pulsoni, Luca Arcaini, Caterina Stelitano, Giovanni Bertoldero, Nicola Cascavilla, Flavia Salvi, Andrés J.M. Ferreri, Massimo Federico

Collection and assembly of data: Stefano Luminari, Martina Manni, Alessandra Dondi, Annalisa Chiarenza, Francesco Merli, Chiara Rusconi, Vittoria Tarantino, Alessandra Tucci, Umberto Vitolo, Sofia Kovalchuk, Emanuele Angelucci, Alessandro Pulsoni, Luca Arcaini, Francesco Angrilli, Gianluca Gaidano, Caterina Stelitano, Giovanni Bertoldero, Nicola Cascavilla, Flavia Salvi, Andrés J.M. Ferreri, Daniele Vallisa, Luigi Marcheselli, Massimo Federico

Data analysis and interpretation: Stefano Luminari, Angela Ferrari, Martina Manni, Alessandra Dondi, Francesco Merli, Vittoria Tarantino, Sofia Kovalchuk, Luca Arcaini, Andrés J.M. Ferreri, Luigi Marcheselli, Massimo Federico

Manuscript writing: All authors

Final approval of manuscript: All authors

Accountable for all aspects of the work: All authors

\section{REFERENCES}

1. Anderson JR, Armitage JO, Weisenburger DD: Epidemiology of the non-Hodgkin's lymphomas: Distributions of the major subtypes differ by geographic locations-Non-Hodgkin's Lymphoma Classification Project. Ann Oncol 9:717-720, 1998

2. Casulo C, Burack WR, Friedberg JW: Transformed follicular non-Hodgkin lymphoma. Blood 125: 40-47, 2015

3. Fisher RI, LeBlanc M, Press OW, et al: New treatment options have changed the survival of patients with follicular lymphoma. J Clin Oncol 23: 8447-8452, 2005

4. Schulz H, Bohlius JF, Trelle S, et al: Immunochemotherapy with rituximab and overall survival in patients with indolent or mantle cell lymphoma: A systematic review and meta-analysis. J Natl Cancer Inst 99:706-714, 2007

5. Vidal L, Gafter-Gvili A, Salles G, et al: Rituximab maintenance for the treatment of patients with follicular lymphoma: An updated systematic review and meta-analysis of randomized trials. J Natl Cancer Inst 103:1799-1806, 2011

6. Salles G, Seymour JF, Offner F, et al: Rituximab maintenance for 2 years in patients with high tumour burden follicular lymphoma responding to rituximab plus chemotherapy (PRIMA): A phase 3, randomised controlled trial. Lancet 377:42-51, 2011 [Erratum: Lancet 377:1154, 2011]

7. Federico M, Luminari S, Dondi $A$, et al: R-CVP versus $\mathrm{R}-\mathrm{CHOP}$ versus R-FM for the initial treatment of patients with advanced-stage follicular lymphoma: Results of the FOLL05 trial conducted by the Fondazione Italiana Linfomi. J Clin Oncol 31:1506-1513, 2013 [Erratum: J Clin Oncol 32:1095, 2014]
8. Swerdlow SH, Campo E, Harris N, et al (eds): WHO Classification of Tumors of Haematopoietic and Lymphoid Tissues. Lyon, France, IARC Press, 2008

9. Barosi G, Carella A, Lazzarino $M$, et al: Management of nodal indolent (non marginal-zone) nonHodgkin's lymphomas: Practice guidelines from the Italian Society of Hematology, Italian Society of Experimental Hematology and Italian Group for Bone Marrow Transplantation. Haematologica 90:1236-1257, 2005

10. Luminari S, Biasoli I, Versari A, et al: The prognostic role of post-induction FDG-PET in patients with follicular lymphoma: A subset analysis from the FOLL05 trial of the Fondazione Italiana Linfomi (FIL). Ann Oncol 25:442-447, 2014

11. Galimberti $S$, Luminari $S$, Ciabatti $E$, et al: Minimal residual disease after conventional treatment significantly impacts on progression-free survival of patients with follicular lymphoma: The 
FIL FOLL05 trial. Clin Cancer Res 20:6398-6405 2014

12. Gooley TA, Leisenring $W$, Crowley $J$, et al: Estimation of failure probabilities in the presence of competing risks: New representations of old estimators. Stat Med 18:695-706, 1999

13. Gray RJ: A class of K-sample tests for comparing the cumulative incidence of a competing risk. Ann Stat 16:1141-1154, 1988

14. Fine JP, Gray RJ: A proportional hazards model for the subdistribution of a competing risk. J Am Stat Assoc 94:496-509, 1999

15. Bachy E, Houot R, Morschhauser F, et al: Long-term follow up of the FL2000 study comparing CHVP-interferon to CHVP-interferon plus rituximab in follicular lymphoma. Haematologica 98:1107-1114, 2013

16. Herold M, Scholz CW, Rothmann F, et al: Longterm follow-up of rituximab plus first-line mitoxantrone, chlorambucil, prednisolone and interferon-alpha as maintenance therapy in follicular lymphoma. J Cancer Res Clin Oncol 141:1689-1695, 2015

17. Salles GA, Seymour JF, Feugier $P$, et al: Updated 6 year follow-up of the PRIMA study confirms the benefit of 2-year rituximab maintenance in follicular lymphoma patients responding to frontline immunochemotherapy. Blood 122, 2013 (abstr 509)

18. Tam CS, Seymour JF, Prince HM, et al: Treatment-related myelodysplasia following fludarabine combination chemotherapy. Haematologica 91 1546-1550, 2006

19. Hirayama $Y$, Ishitani $K$, Ota $S$, et al: Long-term survey of survival time, histological transformation, and secondary malignancies in Japanese patients with advanced-stage follicular lymphoma in the rituximab era: Hokkaido Hematology Study Group. Int J Hematol 100:281-289, 2014

20. McLaughlin $P$, Estey $E$, Glassman $A$, et al: Myelodysplasia and acute myeloid leukemia following therapy for indolent lymphoma with fludarabine, mitoxantrone, and dexamethasone (FND) plus ritux imab and interferon alpha. Blood 105:4573-4575, 2005

21. Beiggi $S$, Johnston JB, Seftel MD, et al: Increased risk of second malignancies in chronic lymphocytic leukaemia patients as compared with follicular lymphoma patients: A Canadian population-based study. Br J Cancer 109:1287-1290, 2013

22. Waterman J, Rybicki L, Bolwell B, et al: Fludarabine as a risk factor for poor stem cell harvest, treatment-related MDS and AML in follicular lymphoma patients after autologous hematopoietic cell transplantation. Bone Marrow Transplant 47:488-493 2012

23. Sacchi $S$, Marcheselli $L$, Bari $A$, et al: Secondary malignancies after treatment for indolent nonHodgkin's lymphoma: A 16-year follow-up study. Haematologica 93:398-404, 2008

24. Magnano L, Montoto S, González-Barca E, et al: Long-term safety and outcome of fludarabine, cyclophosphamide and mitoxantrone (FCM) regimen in previously untreated patients with advanced follicular lymphoma: 12 years follow-up of a phase 2 trial. Ann Hematol 96:639-646, 2017

25. Nastoupil LJ, McLaughlin P, Feng L, et al: High ten-year remission rates following rituximab, fludarabine, mitoxantrone and dexamethasone (R-FND) with interferon maintenance in indolent lymphoma: Results of a randomized study. Br J Haematol 177 263-270, 2017

26. Thieblemont $C$, Tilly $H$, Gomes da Silva $M$, et al: Lenalidomide maintenance compared with placebo in responding elderly patients with diffuse large B-cell lymphoma treated with first-line rituximab plus cyclophosphamide, doxorubicin, vincristine, and prednisone. J Clin Oncol 35:2473-2481, 2017

27. Flinn IW, van der Jagt $R$, Kahl BS, et al: Randomized trial of bendamustine-rituximab or R-CHOP/ $\mathrm{R}-\mathrm{CVP}$ in first-line treatment of indolent NHL or MCL: The BRIGHT study. Blood 123:2944-2952, 2014

28. Tao L, Clarke CA, Rosenberg AS, et al: Subsequent primary malignancies after diffuse large B-cell lymphoma in the modern treatment era. $\mathrm{Br} J$ Haematol 178:72-80, 2017

29. Kuruvilla J, Assouline S, Hodgson D, et al: A Canadian evidence-based guideline for the first-line treatment of follicular lymphoma: Joint consensus of the Lymphoma Canada Scientific Advisory Board. Clin Lymphoma Myeloma Leuk 15:59-74, 2015

30. Cheson BD, Brugger W, Damaj G, et al: Optimal use of bendamustine in hematologic disorders: Treatment recommendations from an international consensus panel-An update. Leuk Lymphoma 57: 766-782, 2016

31. Rummel MJ, Niederle N, Maschmeyer G, et al: Bendamustine plus rituximab versus $\mathrm{CHOP}$ plus rituximab as first-line treatment for patients with indolent and mantle-cell lymphomas: An open-label, multicentre, randomised, phase 3 non-inferiority trial. Lancet 381:1203-1210, 2013

32. Rummel MJ, Maschmeyer G, Ganser A, et al: Bendamustine plus rituximab (B-R) versus $\mathrm{CHOP}$ plus rituximab (CHOP-R) as first-line treatment in patients with indolent lymphomas: Nine-year updated results from the StiL NHL1 study. J Clin Oncol 35 2017 (suppl; abstr 7501)

33. Flinn I, Van der Jagt $R$, Chang JE, et al: First-line treatment of $\mathrm{NNHL}$ or $\mathrm{MCL}$ patients with $\mathrm{BR}$ or R-CHOP/R-CVP: Results of the BRIGHT 5-year followup study. J Clin Oncol 35, 2017 (suppl; abstr 7500)

34. Marcus RE, Davies $A J$, Ando $K$ et al: Obinutuzumab-based induction and maintenance prolongs progression-free survival (PFS) in patients with previously untreated follicular lymphoma: Primary results of the randomized phase 3 GALLIUM study. Blood, 2016 (abstr 6)

35. Montoto S, Davies AJ, Matthews J, et al: Risk and clinical implications of transformation of follicular lymphoma to diffuse large B-cell lymphoma. J Clin Oncol 25:2426-2433, 2007

36. Al-Tourah AJ, Gill KK, Chhanabhai $M$, et al: Population-based analysis of incidence and outcome of transformed non-Hodgkin's lymphoma. J Clin Oncol 26:5165-5169, 2008

37. Wagner-Johnston ND, Link BK, Byrtek $M$ et al: Outcomes of transformed follicular lymphoma in the modern era: A report from the National LymphoCare Study (NLCS). Blood 126:851-857, 2015

38. Sarkozy C, Trneny M, Xerri L, et al: Risk factors and outcomes for patients with follicular lymphoma who had histologic transformation after response to first-line immunochemotherapy in the PRIMA trial. $\mathrm{J}$ Clin Oncol 34:2575-2582, 2016

39. Federico $M$, Caballero $D$, Marcheselli $L$, et al: The risk of transformation of follicular lymphoma "transformed" by rituximab: The ARISTOTLE study promoted by the European Lymphoma Institute. Hematol Oncol 35, 2017 (abstr 105)

\section{Affiliations}

Stefano Luminari, Angela Ferrari, and Francesco Merli, Azienda Ospedaliera Arcispedale Santa Maria Nuova-Istituto di Ricovero e Cura a Carattere Scientifico (IRCCS), Reggio Emilia; Stefano Luminari, Martina Manni, Alessandra Dondi, Vittoria Tarantino, Luigi Marcheselli, and Massimo Federico, University of Modena and Reggio Emilia, Modena, Italy; Annalisa Chiarenza, Azienda Ospedaliera Universitaria Policlinico-Vittorio Emanuele, University of Catania, Catania; Chiara Rusconi, Azienda Socio Sanitaria Territoriale (ASST) -Grande Ospedale Metropolitano Niguarda; Andrés J.M. Ferreri, IRCCS San Raffaele Scientific Institute, Milano; Alessandra Tucci, ASST-Spedali Civili di Brescia, Brescia; Umberto Vitolo, Città della Salute e della Scienza University and Hospital, Torino; Sofia Kovalchuk, University of Florence, Florence; Emanuele Angelucci, Ospedale Policlinico San Martino, Genova; Alessandro Pulsoni, Sapienza University of Rome, Rome; Luca Arcaini, Fondazione IRCCS Policlinico San Matteo, Pavia; Francesco Angrilli, Spirito Santo Hospital, Pescara; Gianluca Gaidano, University of Eastern Piedmont, Novara; Caterina Stelitano, Grande Ospedale Metropolitano Bianchi-Melacrino-Morelli, Reggio Calabria; Giovanni Bertoldero, Ospedale di Mirano, Mirano; Nicola Cascavilla, IRCCS Casa Sollievo della Sofferenza Hospital, San Giovanni Rotondo; Flavia Salvi, Ospedale SS Antonio e Biagio, Alessandria; and Daniele Vallisa, Guglielmo da Saliceto Hospital, Piacenza, Italy.

\section{Support \\ Supported in part by grants from the Associazione Angela Serra per la Ricerca sul Cancro, Modena, Italy.}

Prior Presentation

Presented orally at the 14th International Conference on Malignant Lymphomas, Lugano, Switzerland, June 14-17, 2017. 


\section{AUTHORS' DISCLOSURES OF POTENTIAL CONFLICTS OF INTEREST}

Long-Term Results of the FOLL05 Trial Comparing R-CVP Versus R-CHOP Versus R-FM for the Initial Treatment of Patients With Advanced-Stage Symptomatic Follicular Lymphoma

The following represents disclosure information provided by authors of this manuscript. All relationships are considered compensated. Relationships are self-held unless noted. I = Immediate Family Member, Inst = My Institution. Relationships may not relate to the subject matter of this manuscript. For more information about ASCO's conflict of interest policy, please refer to www.asco.org/rwc or ascopubs.org/jco/site/ifc.

\section{Stefano Luminari}

Consulting or Advisory Role: Roche, Celgene, Gilead Sciences, Sandoz Travel, Accommodations, Expenses: Celgene, Janssen Pharmaceuticals, Takeda Pharmaceuticals

\section{Angela Ferrari}

No relationship to disclose

\section{Martina Manni}

No relationship to disclose

Alessandra Dondi

No relationship to disclose

\section{Annalisa Chiarenza}

No relationship to disclose

\section{Francesco Merli}

Consulting or Advisory Role: Mundipharma, Roche, Celgene, Janssen Pharmaceuticals, Takeda Pharmaceuticals, Gilead Sciences

Research Funding: Roche

Travel, Accommodations, Expenses: Takeda Pharmaceuticals

Chiara Rusconi

Consulting or Advisory Role: Takeda Pharmaceuticals, Italfarmaco, Janssen Pharmaceuticals, Sanofi, Celgene

Travel, Accommodations, Expenses: TEVA Pharmaceuticals Industries, Takeda Pharmaceuticals, Roche

Vittoria Tarantino

No relationship to disclose

\section{Alessandra Tucci}

Consulting or Advisory Role: TEVA Pharmaceuticals Industries Travel, Accommodations, Expenses: Roche

\section{Umberto Vitolo}

Consulting or Advisory Role: Roche, Janssen Pharmaceuticals, Celgene Speakers' Bureau: Roche, Celgene, Janssen Pharmaceuticals, Gilead Sciences, Takeda Pharmaceuticals

Sofia Kovalchuk

No relationship to disclose

Emanuele Angelucci

Honoraria: Novartis

Consulting or Advisory Role: Amgen, Roche, Novartis, Celgene

Travel, Accommodations, Expenses: Jazz Pharmaceuticals

\section{Alessandro Pulsoni}

No relationship to disclose

\section{Luca Arcaini}

Consulting or Advisory Role: Celgene, Roche, Bayer HealthCare Pharmaceuticals, Gilead Sciences, Sandoz

Research Funding: Gilead Sciences

Travel, Accommodations, Expenses: Celgene, Gilead Sciences

Francesco Angrilli

No relationship to disclose

Gianluca Gaidano

Honoraria: Roche, Amgen, Janssen Pharmaceuticals, Gilead Sciences, MorphoSys

Consulting or Advisory Role: Roche, Amgen, Janssen Pharmaceuticals, Gilead Sciences, MorphoSys

Speakers' Bureau: Roche, Janssen Pharmaceuticals, Gilead Sciences Travel, Accommodations, Expenses: Janssen Pharmaceuticals

\section{Caterina Stelitano}

No relationship to disclose

Giovanni Bertoldero

No relationship to disclose

Nicola Cascavilla

No relationship to disclose

Flavia Salvi

No relationship to disclose

Andrés J.M. Ferreri

No relationship to disclose

Daniele Vallisa

Travel, Accommodations, Expenses: Roche, Janssen Pharmaceuticals

Luigi Marcheselli

No relationship to disclose

Massimo Federico

No relationship to disclose 


\section{Appendix}

List of investigators participating in the study: M. Ladetto (Alessandria), M. Marchetti (Asti), M. Spina (Istituto di Ricovero e Cura a Carattere Scientifico [IRCCS] Aviano Oncologia), A. Guarini (IRCCS Bari), A. Conconi (Ematologia Biella), G. Rossi (Ematologia Brescia), F. Di Raimondo (Ematologia Catania Ferrarotto), P. Guglielmo (Catania Nesima), R. Cantaffa (Catanzaro), R. Freilone (Ciriè), R. Centurioni (Civitanova Marche), F. Morabito (Cosenza), L. Rigacci (Firenze), N. Acquarone (Genova Galliera), E. Angelucci (Genova San Martino), N. Di Renzo (Lecce), P. Ferrando (Lecco), A. Fragasso (Matera), D. Mannina (Messina Papardo), C. Musolino (Messina Policlinico), D. Pedretti (Milano Fatebenefratelli), A. Santoro (Milano Humanitas), L. Baldini (Milano Marcora), E. Morra (Milano Niguarda), L. Tedeschi (Milano San Carlo Borromeo), D. Ferrari (Milano San Paolo), A.J.M. Ferreri (Milano San Raffaele), G. Bertoldero (Mirano), M. Federico (Modena), V. Mettivier (Napoli Cardarelli), M. Barone (Nocera Pagani), G. Gaidano (Novara), L. Arcaini (Pavia), B. Falini (Perugia), F. Angrilli (Pescara Ematologia), F. Lombardo (Pescara Oncologia), D. Vallisa (Piacenza), M. Petrini (Pisa), C. Stelitano (Reggio Calabria), F. Merli (Reggio Emilia), P. Musto (Rionero), O. Annibali (Roma Campus Biomedico), S. Hohaus (Roma Cattolica), F. Palombi (Roma Ifo Regina Elena), A. Pulsoni (Roma La Sapienza), M.C. Cox (Roma Sant'Andrea), M. Cantonetti (Roma Torvergata), N. Cascavilla (San Giovanni Rotondo), G. Partesotti (Sassuolo), M. Liberati (Terni), M. Aglietta (Torino Candiolo), U. Vitolo (Torino Molinette), V. Pavone (Tricase), S. Passamonti (Varese), and R. Bassan (Venezia Mestre). 